\title{
Estabilización y desestabilización por adsorción sobre coloides modelo de Triton X-100
}

\author{
M.S. ROMERO-CANO ${ }^{1}$, A. MARTÍN-RODRÍGUEZ ${ }^{2}$ Y F.J. DE LAS NIEVES ${ }^{1}$ \\ ${ }^{1}$ Grupo de Física de Fluidos Complejos. Departamento de Física Aplicada. Universidad de Almería. 04120 Almería. \\ ${ }^{2}$ Grupo de Física de Fluidos y Biocoloides. Departamento de Física Aplicada. Universidad de Granada. 18071 Granada.
}

\begin{abstract}
Se ha estudiado la estabilidad coloidal de partículas de poliestireno con diferente funcionalidad superficial (sulfato y carboxilo) después de la adsorción de Triton X-100. El grado de recubrimiento y el $\mathrm{pH}$ del medio fueron variables fundamentales en el estudio. Se han encontrado tres tipos diferentes de estabilización coloidal que pueden clasificarse en función de la relación entre la energía de interacción atractiva de van der Waals $\left(V_{A}\right)$ y la energía de interacción repulsiva de origen estérico $\left(V_{S}\right)$ : electrostático $\left(V_{S}=0\right)$, electroestérico $\left(V_{S}<\left|V_{A}\right|\right)$ y estérico $\left(V_{S}>\left|V_{A}\right|\right)$. La diferencia fundamental entre estos tres tipos de estabilización es la respuesta frente a la concentración de electrolito: los dos primeros son sensibles a la adición del mismo mientras que el último no lo es. Adicionalmente se han encontrado fenómenos de desestabilización, no predichos por la teoría DLVO extendida, los cuales dependen de la funcionalidad superficial, del grado de recubrimiento y del $\mathrm{pH}$ del medio de dispersión.
\end{abstract}

Palabras clave: Estabilidad coloidal, estabilidad estérica, coloides poliméricos.

\section{Stabilization and desestabilization of model colloids after adsorption of Triton X-100}

The colloidal stability of polystyrene particles with different surface functional groups (sulfate and carboxyl) has been studied after adsorption of Triton X-100. The coverage degree and the medium $\mathrm{pH}$ were the main factors in this study. Three different types of colloidal stabilization were found, which can be clasified as a function of the relationship between the atractive interaction energy $\left(\mathrm{V}_{\mathrm{A}^{\prime}}\right.$, Van der Waals type) and the steric interaction energy $\left(\mathrm{V}_{\mathrm{S}}\right.$, repulsive type): electrostatic $\left(\mathrm{V}_{\mathrm{S}}=0\right)$, electrosteric $\left(\mathrm{V}_{\mathrm{S}}<\left|\mathrm{V}_{\mathrm{A}}\right|\right)$, and steric $\left(\mathrm{V}_{\mathrm{S}}>\left|\mathrm{V}_{\mathrm{A}}\right|\right)$. The main difference between these stabilization types is the response against the electrolyte concentration: the first and second cases are affected by electrolyte addition, while the third one does not.

Key words: Colloidal stability; steric stability: polymer colloids.

\section{INTRODUCCIÓN}

Hoy en día, el número de opciones para estabilizar una dispersión coloidal está fuertemente limitado, existiendo dos vías generales mediante las cuales se puede proporcionar estabilidad coloidal: estabilización electrostática y estabilización estérica.

Según la teoría DLVO (1-2), la estabilidad de una dispersión electrostáticamente estabilizada viene determinada por la superposición de la energía de atracción de London van der Waals $\left(V_{A}\right)$ y por la energía repulsiva electrostática $\left(V_{R}\right)$. Mientras $V_{A}$ no es sensible a la concentración de electrolito, $V_{R}$ si lo es, y por lo tanto la estabilidad de estas dispersiones coloidales puede ser controlada mediante el cambio de la concentración de electrolito del medio.

Adicionalmente, cuanto se adsorbe un surfactante no-iónio sobre las partículas coloidales, éste puede actuar como agente estabilizador (3). En este caso la estabilidad coloidal es proporcionada por la combinación de efectos electrostáticos y estéricos. Una de las ventajas que presenta el mecanismo de estabilización de tipo estérico es su insensibilidad ante la adición de electrolito, como indican los potenciales de interacción estérica propuestos por Vincent et al. (4-5). La teoría de estabilidad coloidal que incluye los factores estéricos, denominada DLVO extendida, considera que la energía potencial total de interacción viene dada por la superposición de las tres contribuciones anteriormente mencionadas: $\mathrm{V}=\mathrm{V}_{\mathrm{A}}+\mathrm{V}_{\mathrm{R}}+$ $\mathrm{V}_{\mathrm{S}}$.

Es posible introducir una clasificación de las dispersiones coloidales basada en su respuesta a la adición de electrolito, la cual está directamente relacionada con la magnitud de la energía de interacción estérica.

i. Sensible a la adición de electrolito: estabilización electrostática $\left(\mathrm{V}_{\mathrm{S}}=0\right)$ y electroestérica $\left(\mathrm{V}_{\mathrm{S}}<\left|\mathrm{V}_{\mathrm{A}}\right|\right)$.

ii. Insensible a la adición de electrolito: estabilización estérica $\left(\mathrm{V}_{\mathrm{S}}>\left|\mathrm{V}_{\mathrm{A}}\right|\right)$.

En este trabajo se han utilizado dos tipos de látex (sulfato y carboxilo) y un surfactante no-iónico modelo (Triton X-100) como agente estabilizador. Controlando el $\mathrm{pH}$ del medio y el grado de recubrimiento de los complejos látex-surfactante preparados, podremos observar los tres tipos de estabilización coloidal anteriormente mencionados.

\section{PROCEDIMIENTO EXPERIMENTAL}

\subsection{Materiales}

Las características de los materiales utilizados en este estudio, así como los detalles de la preparación de los complejos látex-surfactante aparecen en trabajos previos (6-7). En con- 
creto, las partículas coloidales utilizadas en este trabajo son dos látex de poliestireno con funcionalidad sulfato y carboxilo. La densidad de carga superficial, obtenida por valoración conductimétrica, es de $-(3.2 \pm 0.5) \mu \mathrm{C} / \mathrm{cm}^{2}$ para el látex sulfato y oscila entre 0 y $-(21 \pm 3) \mu \mathrm{C} / \mathrm{cm}^{2}$ para el látex carboxilo. Por lo que respecta al surfactante utilizado, Triton X-100, los análisis de espectroscopía de masas indican una gran pureza teniendo un índice de polidispersidad $\left(\left\langle\mathrm{M}_{\mathrm{w}}>/<\mathrm{M}_{\mathrm{n}}\right\rangle\right)$ de 1.031. Los complejos látex-surfactante se preparan mezclando cantidades adecuadas de estos dos componentes en el medio que se desee (6-7). Este sistema se mantiene con ligera agitación a $(25 \pm 0.1)^{\circ} \mathrm{C}$ durante $24 \mathrm{~h}$.

\section{RESULTADOS Y DISCUSIÓN}

Se ha estudiado la estabilidad coloidal de complejos látexsurfactante con diferente grado de recubrimiento a dos $\mathrm{pH}$ extremos. Controlando estas dos variables podemos introducir al sistema en diferentes regiones de estabilización coloidal caracterizadas por diferentes combinaciones de energías de interacción repulsivas de origen eléctrico y estérico.

En la Figura 1 se muestra la variación de la absorbancia $(\mathrm{dAb} / \mathrm{dt})$ respecto a la concentración de $\mathrm{NaCl}$ del látex sulfato (PS-S) con diferente cantidad de Triton X-100 adsorbida. Para este sistema en concreto no se encontró ninguna dependencia entre la estabilidad coloidal de los complejos PS-S-TX100 y el $\mathrm{pH}$, resultado que era de esperar debido al carácter ácido fuerte de los grupos superficiales. Analizando esta figura se observa como la presencia del surfactante en la superficie de las partículas provoca una mejora en la estabilidad coloidal del sistema, debido a la aparición de una energía de interacción repulsiva de origen estérico. La mejora encontrada en la estabilidad coloidal de la dispersión depende del grado de recubrimiento llegándose a una estabilización total para los complejos con máximo recubrimiento. En esta transición se observan claramente los tres tipos de estabilización mencionados anteriormente.

Por lo que respecta al látex carboxilo, el comportamiento si resulta $\mathrm{pH}$ dependiente. Las Figuras 2 y 3 muestran la variación de la absorbacia $(\mathrm{dAb} / \mathrm{dt})$ respecto a la concentración de $\mathrm{NaCl}$ del látex carboxilo (PS-C) con diferente cantidad de Triton X-100 adsorbida a pHs 5 y 10, respectivamente. El carácter ácido débil de los grupos carboxilo, proporciona una repulsión electrostática que será máxima a $\mathrm{pH} 10$, donde estos grupos están desprotonados, y mucho menor a $\mathrm{pH} 5$, donde la mayoría de los grupos ácido débil estarán protonados. Globalmente, las tendencias encontradas están en concordancia con los resultados obtenidos para el látex sulfato. Sin embargo, el complejo PS-C-TX100 con mínimo recubrimiento $\left(1.0 \mu \mathrm{mol} / \mathrm{m}^{2}\right)$ presenta una estabilidad coloidal inferior a la del látex sin recubrir. Este resultado no puede ser explicado con las actuales teorías de estabilidad coloidal. El hecho de que este fenómeno inesperado ocurra sólo bajo unas condiciones muy específicas, induce a pensar en la aparición de un mecanismo adicional dependiente de las características fisico-químicas del surfactante, de la superficie de las partículas de látex y del medio de dispersión. La química clásica predice una interacción específica mediante puentes de hidrógeno (8) entre el grupo óxido de etileno, presente en la cadena estabilizadora de la molécula de surfactante, y los grupos carboxilo superficiales. La probabilidad de que esta interacción se produzca a alto recubrimiento de surfactante es pequeña debido al alto grado de compactación de la capa de surfactante, mientras que está favorecida a bajo recubri-

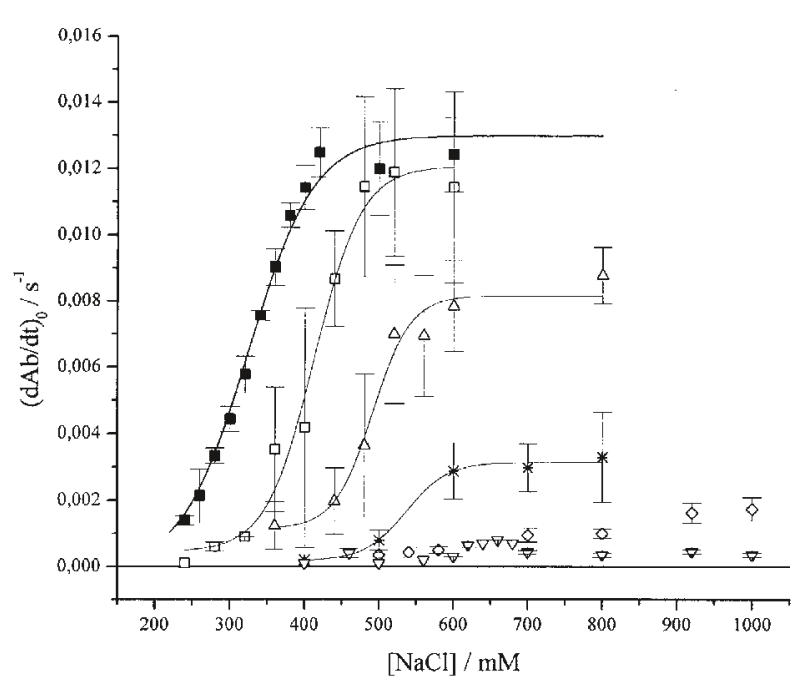

Figura 1. Cinética de agregación del sistema PS-S-TX100 con diferentes recubrimientos: $=$ sin recubrir, $\square=1.0 \mu \mathrm{mol} / \mathrm{m}^{2}, \Delta=1.5 \mu \mathrm{mol} /$ $\mathrm{m}^{2}, *=2.0 \mu \mathrm{mol} / \mathrm{m}^{2}, \diamond=2.5 \mu \mathrm{mol} / \mathrm{m}^{2}, \diamond=3.0 \mu \mathrm{mol} / \mathrm{m}^{2}$.

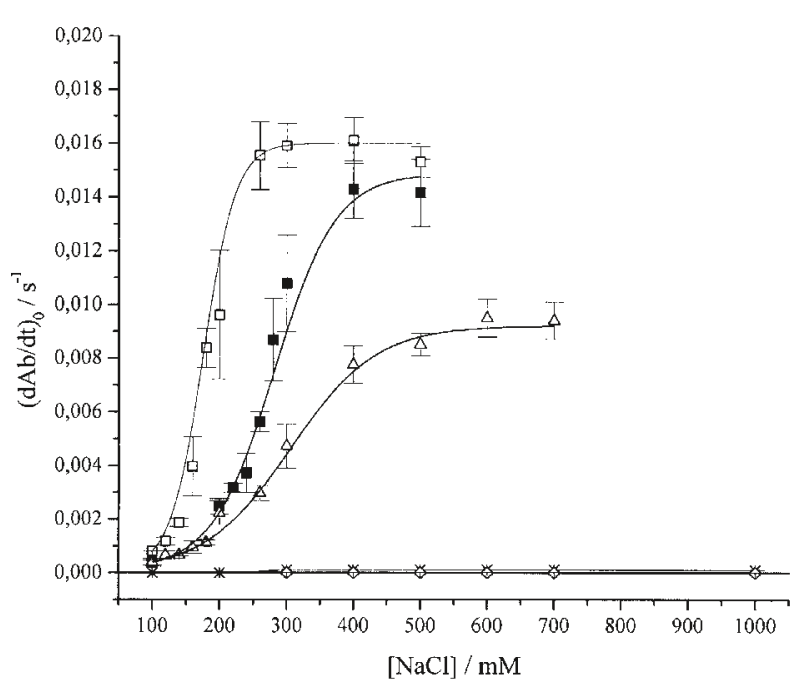

Figura 2. Cinética de agregación del sistema PS-C-TX100 a pH 5 con diferentes recubrimientos: $=\sin$ recubrir, $\square=1.0 \mu \mathrm{mol} / \mathrm{m}^{2}, \Delta=1.5$ $\mu \mathrm{mol} / \mathrm{m}^{2}, *=2.0 \mu \mathrm{mol} / \mathrm{m}^{2}, \nabla=2.5-3-0 \mu \mathrm{mol} / \mathrm{m}^{2}$.

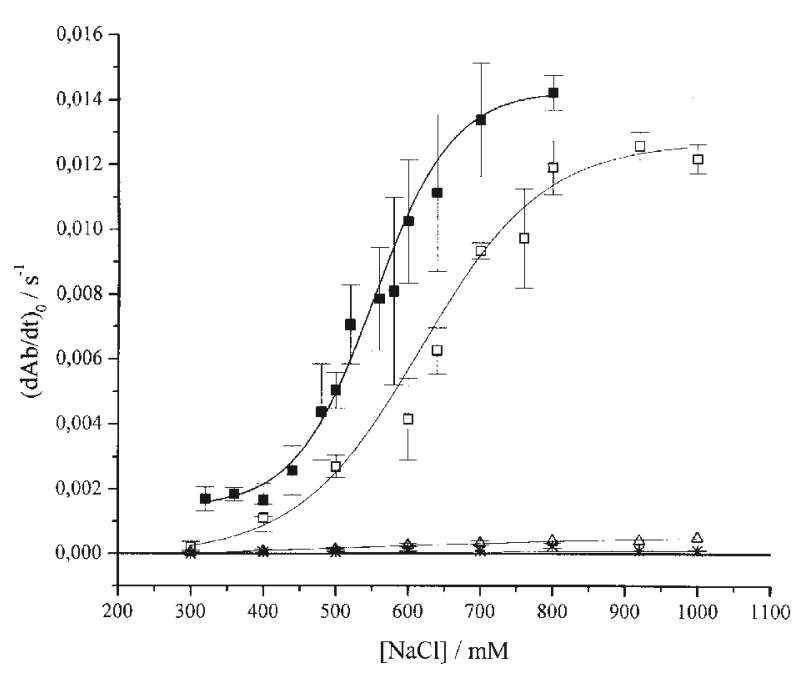

Figura 3. Cinética de agregación del sistema PS-C-TX100 a pH 10 con diferentes recubrimientos: $\boldsymbol{\nabla}=\sin$ recubrir, $\boldsymbol{\square}=1.0 \mu \mathrm{mol} / \mathrm{m}^{2}, \Delta$ $=1.5 \mu \mathrm{mol} / \mathrm{m}^{2}, *=2.0-3.0 \mu \mathrm{mol} / \mathrm{m}^{2}$. 
miento debido a la gran flexibilidad de las partes hidrofílicas de la molécula de Triton X-100. Como consecuencia directa de esta interacción puede producirse un cambio en la conformación de la capa superficial de surfactante, pasando de ser extendida a ser plana. Esto afecta directamente a la magnitud de la energía de interacción repulsiva de origen estérico. Sin embargo, este cambio sólo justifica la disminución de la barrera energética repulsiva, y por consiguiente de la estabilidad coloidal, pero no explica la desestabilización respecto al látex sin recubrir. La clave de este fenómeno la encontramos en trabajos previos donde se caracterizan electrocinéticamente estos sistemas coloidales $(6,9)$. Estos estudios parecen indicar un exceso de carga positiva $\left(\mathrm{H}^{+}\right)$en la interacción $\mathrm{OE}-\mathrm{COOH}$ que actuaría apantallando la carga superficial del látex. Este apantallamiento disminuye la barrera potencial repulsiva electrostática justificando la menor estabilidad coloidal del complejo.

\section{CONCLUSIONES}

Se pueden utilizar los surfactantes no-iónicos como una excelente herramienta para controlar la estabilidad coloidal de una dispersión de partículas de látex. Esta estabilidad se ve mejorada con recubrimiento adecuado. Además, las moléculas de surfactante pueden actuar como agentes desestabilizadores siempre y cuando las características fisico-químicas del surfactante y de la superficie reúnan unos requisitos determinados.

\section{AGRADECIMIENTOS}

Los autores quieren expresar su gratitud a la CICYT (proyecto MAT 96-1035-C03-03).

\section{BIBLIOGRAFÍA}

1. B.V. Derjaguin, L.D. Landau, Acta Physicochim. URSS 14, 633 (1941)

2. E.J.W. Verwey, J.Th.G. Overbeek, en: Theory of Stability of Lyophobic Colloids, Elsevier, Amsterdam 1948.

3. D.H. Napper, en: Polymeric Stabilization of Colloidal Dispersion, Academic Press Inc., London 1983.

4. B. Vincent, P.F. Luckham, F.A. Waite, J. Colloid Interface Sci., 73, 508 (1980).

5. B. Vincent, J. Edwards, S. Emmett, A. Jones, Colloids Surfaces, 18, 261 (1986).

6. M.S. Romero-Cano. Tesis Doctoral. Universidad de Granada, 1998

7. M.S. Romero-Cano, A. Martín-Rodríguez, F.J. de las Nieves. I. Colloid Interface Sci, 227 (2000), en prensa.

8. G.C. Pimentel, A.L. McClellan, in: The Hidrogen Bond, Freeman, San Francisco 1960.

9. M.S. Romero-Cano, A. Martín-Rodríguez, F.J. de las Nieves. En preparación 